\title{
NOTCH reprograms mitochondrial metabolism for proinflammatory macrophage activation
}

\author{
Jun Xu, ${ }^{1,2,3}$ Feng Chi, ${ }^{1,2}$ Tongsheng Guo, ${ }^{4}$ Vasu Punj, ${ }^{5}$ W.N. Paul Lee, ${ }^{6}$ Samuel W. French, ${ }^{1,7}$ and Hidekazu Tsukamoto ${ }^{1,2,3}$ \\ ${ }^{1}$ The Southern California Research Center for ALPD and Cirrhosis, Los Angeles, California, USA. ²Department of Pathology, Keck School of Medicine of the University of Southern California, Los Angeles, \\ California, USA. ${ }^{3}$ Department of Veterans Affairs, Greater Los Angeles Healthcare System, Los Angeles, California, USA. ${ }^{4}$ Center for Clinical Diagnostic, Beijing 302 Hospital, Beijing, China. \\ ${ }^{5}$ Keck School of Medicine of the University of Southern California, Los Angeles, California, USA. ${ }^{6}$ Division of Pediatric Endocrinology, LA Biomedical Research Institute, Torrance, California, USA. \\ `Department of Pathology and Laboratory Medicine, Harbor-UCLA Medical Center, Torrance, California, USA.
}

\begin{abstract}
Metabolic reprogramming is implicated in macrophage activation, but the underlying mechanisms are poorly understood. Here, we demonstrate that the NOTCH1 pathway dictates activation of M1 phenotypes in isolated mouse hepatic macrophages (HMacs) and in a murine macrophage cell line by coupling transcriptional upregulation of M1 genes with metabolic upregulation of mitochondrial oxidative phosphorylation and ROS (mtROS) to augment induction of M1 genes. Enhanced mitochondrial glucose oxidation was achieved by increased recruitment of the NOTCH1 intracellular domain (NICD1) to nuclear and mitochondrial genes that encode respiratory chain components and by NOTCH-dependent induction of pyruvate dehydrogenase phosphatase 1 ( $P d p 1$ ) expression, pyruvate dehydrogenase activity, and glucose flux to the TCA cycle. As such, inhibition of the NOTCH pathway or Pdp1 knockdown abrogated glucose oxidation, mtROS, and M1 gene expression. Conditional NOTCH1 deficiency in the myeloid lineage attenuated HMac M1 activation and inflammation in a murine model of alcoholic steatohepatitis and markedly reduced lethality following endotoxin-mediated fulminant hepatitis in mice. In vivo monocyte tracking further demonstrated the requirement of NOTCH1 for the migration of blood monocytes into the liver and subsequent $\mathrm{M} 1$ differentiation. Together, these results reveal that NOTCH1 promotes reprogramming of mitochondrial metabolism for M1 macrophage activation.
\end{abstract}

\section{Introduction}

In response to environment cues, innate monocytes or macrophages (Macs) undergo proinflammatory (M1) or alternative (M2) activation. M1 Macs, induced by Th1 cytokines and bacterial LPS, mediate host defense against microorganisms; whereas M2 Macs, under the influence of Th2 cytokines, render antiinflammatory effects and promote wound healing (1). Besides distinct functions and gene expression profiles of M1 and M2 Macs, they exhibit contrasted metabolic activities (2). M1 Macs are associated with increased glycolysis and production of NO from arginine by inducible NO synthase (NOS2). On the other hand, the M2 Macs rely on fatty acid oxidation and metabolize arginine through arginase 1 (ARG1) (2). These studies suggest the importance of metabolic reprogramming in controlling the fate of Macs, as seen in the fate determination of other cell types (3). However, the molecular mechanisms of the metabolic reprogramming and its causal relation with $\mathrm{M} 1$ activation are unknown.

The highly conserved NOTCH signaling pathway integrates environmental cues and specifies cell fate during development (4). NOTCH activation is initiated by the ligand interaction with NOTCH (5) and by TLR4 activation with LPS in Macs (6, 7). Once activated, NOTCH intracellular domain (NICD) is

Authorship note: Jun Xu and Feng Chi contributed equally to this work.

Conflict of interest: The authors have declared that no conflict of interest exists.

Submitted: April 7, 2014; Accepted: February 9, 2015.

Reference information: J Clin Invest. 2015;125(4):1579-1590. doi:10.1172/JCI76468. liberated by $\gamma$-secretase and translocates to nucleus, in which it binds to the CBF-1/suppressor of hairless/Lag1-containing (CSL-containing) repressor complex on DNA and converts it to an activator complex (5). In Macs, NOTCH activation leads to enhanced M1 gene expression and proinflammatory response in vitro $(6,8)$. Inhibition of NOTCH by the $\gamma$-secretase inhibitor $\mathrm{N}$-[N-(3,5-difluorophenacetyl)-L-alanyl]-S-phenylglycine $t$-butyl ester (DAPT), Notch silencing $(6,8)$, or genetic ablation of Csl (9) diminishes LPS-stimulated M1 gene expression. The in vivo role of NOTCH-dependent M1 activation in disease models, however, remains elusive. NICD also interacts with HIF-1 $\alpha(10,11)$, which is a master regulator of glycolysis (12) and is implicated in M1 activation $(13,14)$. In tumor cells, NICD overexpression increases glycolytic activity through activation of the PI3K/AKT pathway (15). These findings suggest the potential role of NOTCH in linking Mac metabolism to M1 activation.

Mac M1 activation is implicated in the pathogenesis of chronic inflammatory diseases, such as alcoholic steatohepatitis (ASH) (16), nonalcoholic fatty liver disease (17), and insulin resistance and diabetes (18). Chronic alcohol consumption or obesity due to high-fat diet causes dysbiosis and increased gut permeability to PAMPs such as LPS, which enters portal circulation and activates hepatic Macs (HMacs) in ASH or nonalcoholic fatty liver disease $(16,17)$. We previously reported a mouse model of advanced ASH produced by a combination of high-fat diet overfeeding and alcohol intake (OF+Alc mouse) (19) - a condition often seen in obese alcoholic patients (20-22). In the present study, we used HMacs 
A

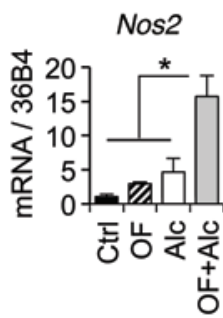

C
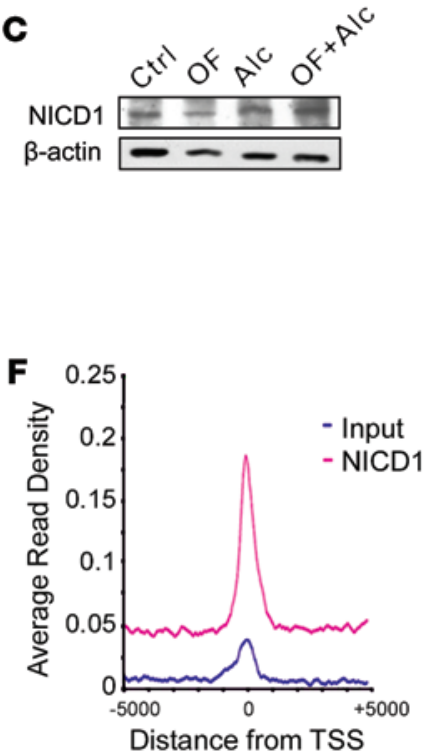
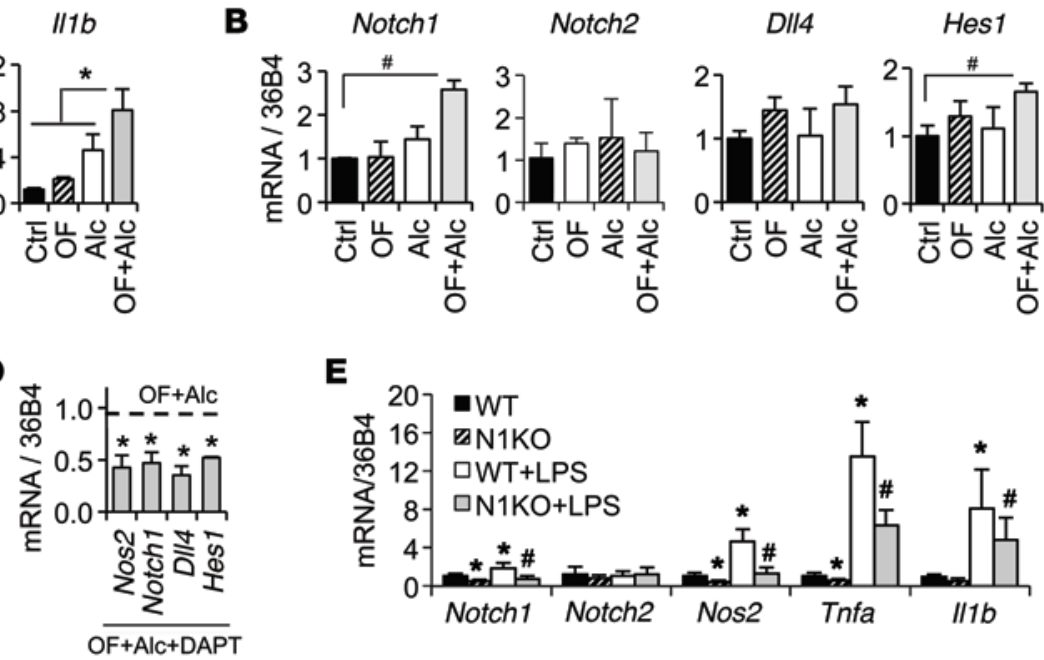

$\mathbf{E}$

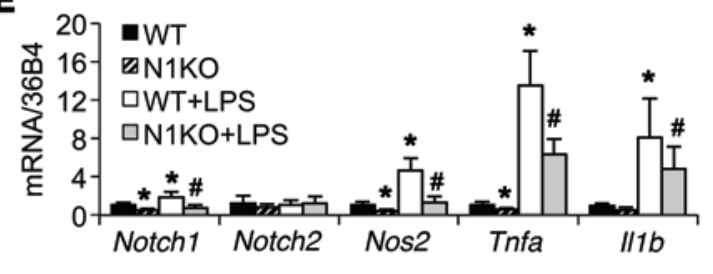

G

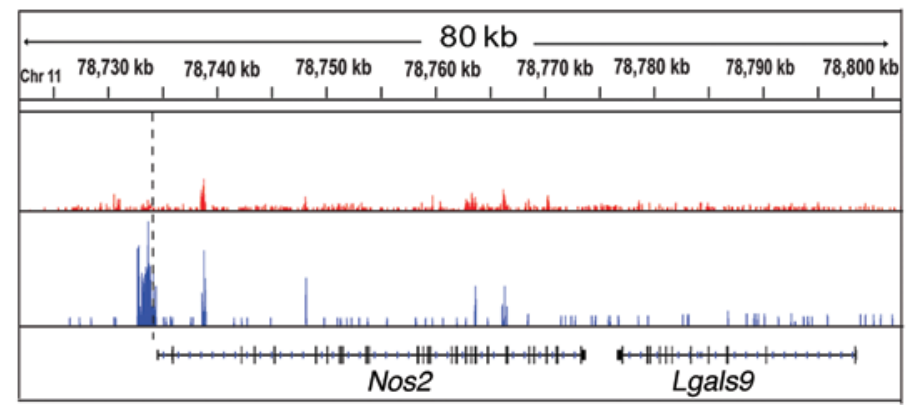

Figure 1. NOTCH-dependent expression of M1 genes. (A) Expression of M1 genes and (B) NOTCH receptors, Notch1 and Notch2, ligand, DII4, and target, Hes1, in HMacs from control (Ctrl), high-fat diet-overfed (OF), alcohol (Alc), or combined OF+Alc mice ( $n=3-5$ per group). ${ }^{*} P<0.01$, ${ }^{\#} P<0.05,1$-way ANOVA. (C) Immunoblot showing increased NICD1 in HMacs from the OF+Alc mice. Results are representative of 4 different experiments. (D) DAPT suppresses gene expression in HMacs from OF+Alc mice ( $n=3-5$ per group). The dashed line refers to the mRNA levels of untreated HMacs, which are set at 1 for comparisons with DAPT-treated HMacs, both of which were isolated from the OF+Alc mice. ${ }^{*} P<0.05$ vs. DAPT-untreated cells, $t$ test. (E) Gene expression in cultured HMacs from WT and Notch1 KO mice treated with or without LPS ( $10 \mathrm{ng} / \mathrm{ml}, 4$ hours) ( $n=6$ per group). ${ }^{*} P<0.05$ vs. WT, ${ }^{\#} P<0.05$ vs. WT+LPS, 1-way ANOVA. (F) Average ChIP enrichment signals are shown over regions spanning $\pm 5 \mathrm{~kb}$ around the transcription start sites (TSSs) of all the mouse genes from UCSC RefSeq database. Blue and red lines indicate the input (no immunoprecipitation) level and NICD1 enrichment by ChIP-seq, respectively. (G) Integrative Genomics Viewer genome browser tracks show the level of NICD1 enrichment near the Nos2 transcription start site in ChIP samples (blue) over input (red). Different genomic coordinates and genome window size for Nos2 (chr11:101,691,391-101,717,344; 26 kb) are shown with $\mathrm{mm} 9$ reference sequence (RefSeq) data. The transcription start site is shown by the dashed line.

isolated from OF+Alc mice and murine Raw 264.7 cells stimulated with LPS with or without IFN- $\gamma$ as in vivo- and in vitro-activated M1 Macs to investigate the role of NOTCH in the metabolic basis of M1 activation. Our results demonstrate that the NOTCH1 pathway is activated and the NOTCH1 intracellular domain (NICD1) is recruited to promoters of Nos 2 and pyruvate dehydrogenase (PDH) phosphatase 1 ( $P d p 1)$ genes to mediate M1 activation and mitochondrial glucose oxidation, respectively. NICD1 is also recruited to mitochondrial DNA (mtDNA), and mtDNA-encoded respiratory chain components are upregulated in the manner dependent on NOTCH. The increased glucose oxidation and mitochondrial respiration lead to enhanced mitochondrial oxidative phosphorylation and ROS (mtROS), which in turn positively regulates M1 genes, establishing what we believe to be a novel positive regulatory loop mediated by NOTCH-dependent reprogramming of mitochondrial metabolism. Inhibition of myeloid NOTCH1 pathway in OF+Alc mice diminishes blood monocyte migration into the liver, HMacs M1 activation, and hepatic inflammation.

\section{Results}

NOTCH1-dependent M1 activation and Nos 2 expression. HMacs isolated from OF+Alc mice (M1 HMacs) had significantly upregulated M1 genes (Nos2, Tnfa, and Il1b) (Figure 1A); modestly increased M2 Arg1; a 4-fold higher Nos2/Arg1 ratio; and a 60\% reduction in M2 Il1O (Supplemental Figure 1A; supplemental material available online with this article; doi:10.1172/JCI76468DS1), depicting a shift toward M1 activation. In these cells, Notch1, but not Notch2, was upregulated, along with the NOTCH target Hes1 (Figure 1B), and NOTCH1 activation was evident by the increased NICD1 protein (Figure 1C). Expression of these genes was suppressed by ex vivo treatment with DAPT (Figure 1D), a $\gamma$-secretase inhibitor that blocks NOTCH activation (23). We also tested the role of NOTCH1 in LPS-stimulated M1 gene induction by examining HMacs isolated from chow-fed myeloid-specific LysM-Cre:Notch1 ${ }^{A / f l}$ (Notch1 KO) mice and littermate WT mice. As shown in Figure 1E, expressions of both basal and LPS-induced M1 genes (Nos2, Tnfa, and Il1b) in WT HMacs were attenuated by Notch1 KO (Figure 1E). 
A

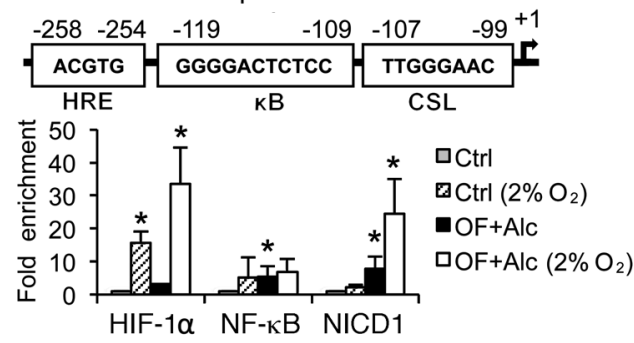

D

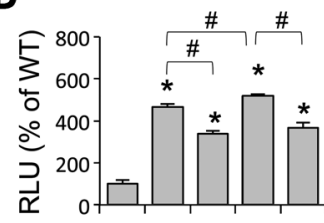

Sh-RNA WT Scr N1 Scr N1 LPS - - - + +
B

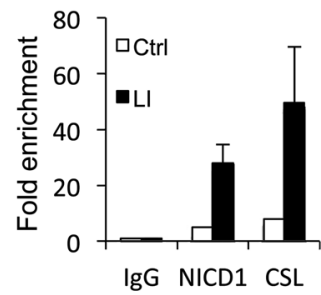

C

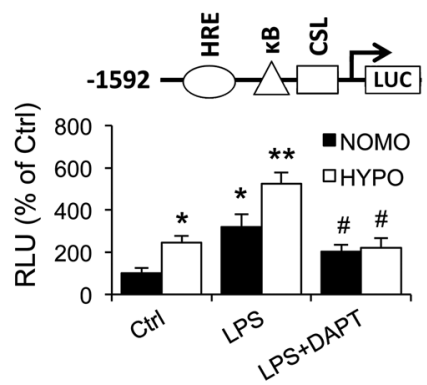

G

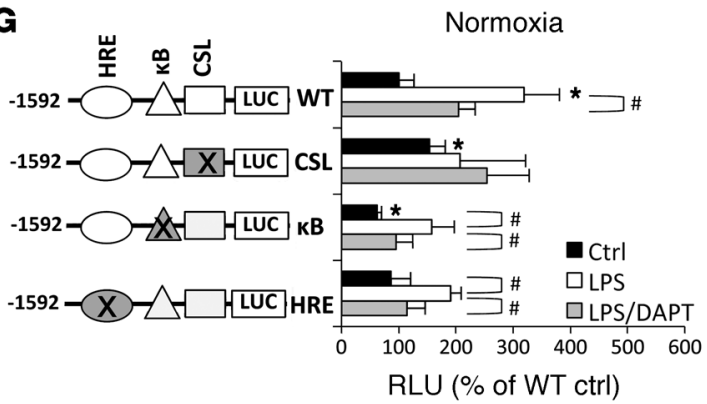

$\mathbf{F}$

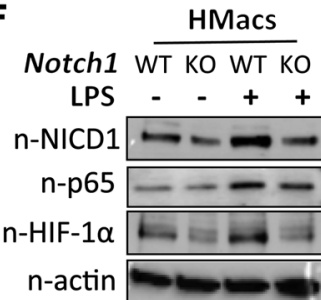

Raw264.7

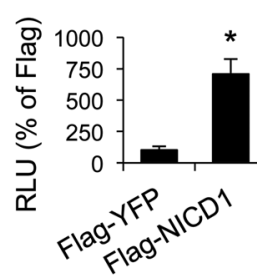

H

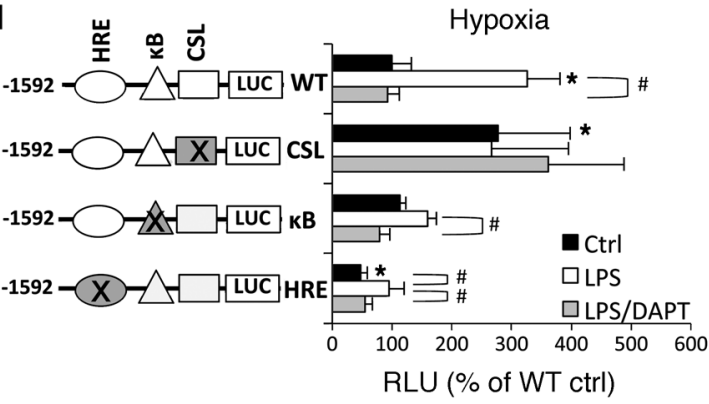

Figure 2. NOTCH directly activates Nos2 transcription. (A) Schematic response elements for HIF-1 $\alpha$ (HRE), NF- $\kappa B$ ( $\kappa B$ ), and NOTCH partner CSL in murine Nos2 promoter. ChIP-qPCR on these sites in cultured HMacs under either 21\% (normoxia) or 2\% (hypoxia) oxygen for 16 hours. Values are fold enrichment relative to control after normalization with IgG $(n=3) .{ }^{*} P<0.05$ vs. control under normoxia, $t$ test. (B) ChIP-qPCR for NICD1 and CSL (RBP-JK) in Raw 264.7 cells treated with or without LI. Results are representative of 2 separate experiments. (C) Luciferase Nos 2 promoter activity in Raw 264.7 cells treated with vehicle, DAPT, or LPS plus DAPT under $21 \%$ (NOMO) or $2 \%$ (HYPO) $\mathrm{O}_{2}$. Values are percentage change of Firefly over Renilla luciferase activity as compared with control under NOMO $(n=4)$. ${ }^{*} P<0.05$ vs. normoxic control, ${ }^{* *} P<0.05$ vs. hypoxic control, ${ }^{*} P<0.05$ with LPS treatment under respective conditions, 2-way ANOVA. (D) Nos2 promoter activity in Raw 264.7 cells transduced with nothing (WT) or scrambled (Scr) or Notch1 (N1) shRNA, with or without LPS for 4 hours $(n=5-8)$. ${ }^{*} P<0.05$ vs. WT, ${ }^{*} P<0.05$, 1-way ANOVA. (E) Nos2 promoter activity in Raw 264.7 cells overexpressing 3xflag-YFP or 3xflag-NICD1

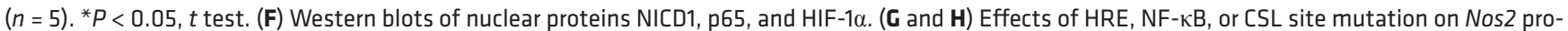
moter activity under (C) normoxia and (H) hypoxia. Raw 264.7 cells transfected with WT or mutant Nos2 promoter luciferase reporters were treated with or without DAPT or LPS for 4 hours $(n=5-8) .{ }^{*} P<0.05$ vs. untreated WT, ${ }^{\#} P<0.05$ between the treatments, $t$ test.

LPS stimulation and myeloid Notch1 KO have minimal effects on the expression of M2 genes (Arg1, Il10, Mrc1, and Chi3l3) (Supplemental Figure 1B). As these primary cells likely represent heterogeneous populations of Macs, we examined LPS-stimulated Raw 264.7 cells as a more homogeneous model of M1 Macs (M1 Raw 264.7 cells) (24). Using these cells, we confirmed the selective upregulation of NOTCH1 and M1 genes (Supplemental Figure 1, C and D). Genome-wide ChIP sequencing (ChIP-seq) analysis of M1 Raw 264.7 cells showed NICD1 enrichments mapping near the transcription initiation sites of 453 nuclear genes (Figure $1 \mathrm{~F})$, of which, 18 genes function in antimicrobial/proinflammatory responses, including M1 genes Nos2 (Figure 1G), Tnf, Il15, and Il17rc (Supplemental Table 1), suggesting a global role of the NOTCH1 pathway in M1 activation. NOTCH1-dependent NOS2 expression was confirmed in the M1 Raw 264.7 cells treated with DAPT (Supplemental Figure 1E) or with lentiviral Notch1-shRNA
(Supplemental Figure 1F). Conversely, NICD1 overexpression increased NOS2 protein expression (Supplemental Figure 1G). Production of NO in M1 Raw 264.7 cells was also diminished by DAPT treatment (Supplemental Figure 1H).

Notch 1 activates Nos 2 transcription. The most proximal region of the mouse Nos 2 promoter (-258/-1) is critical for the activity induced by LPS or LPS plus IFN- $\gamma$ (LI) (25). This region contains the response elements for the NICD partner CSL, NF- $\mathrm{kB}$, and HIF-1 $\alpha$ (Figure 2A). NICD1 binding to the Nos 2 promoter detected by ChIP-seq (Figure 1G) was confirmed by ChIP-quantitative PCR (ChIP-qPCR), which shows enrichments of NICD1 and NF-kB at their respective elements in M1 HMacs compared with the cells from the controls (Figure 2A). Exposure of the cells to hypoxia, the condition commonly seen in ASH $(26,27)$, further increased the enrichments of NICD1 and HIF-1 $\alpha$ (Figure 2A). In Raw 264.7 cells, ChIP-qPCR showed both NICD1 and CSL (RBP-Jк) binding 
A
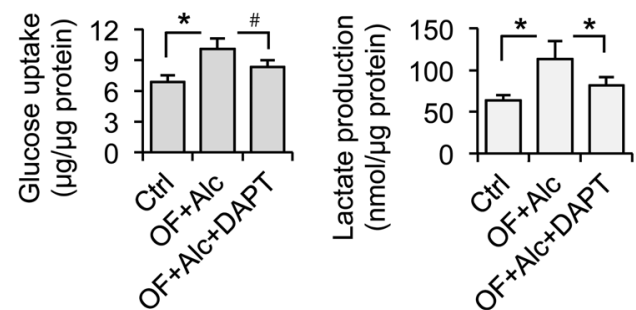

B

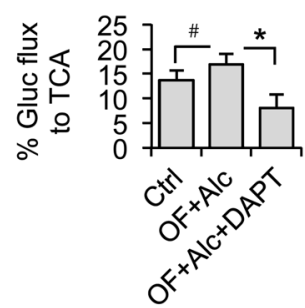

C

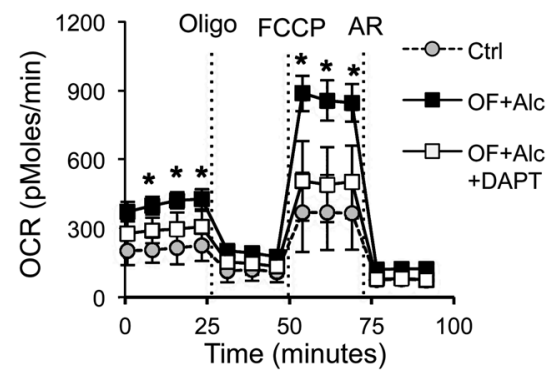

D

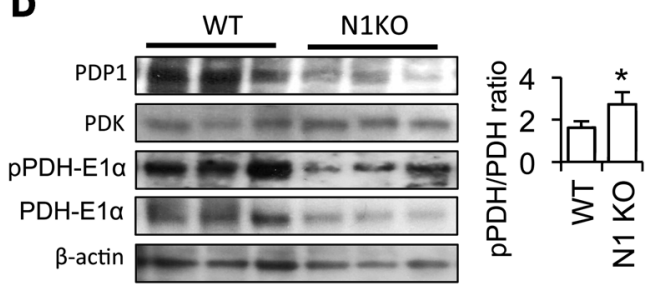

E

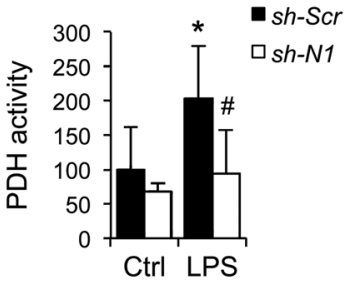

$\mathbf{F}$

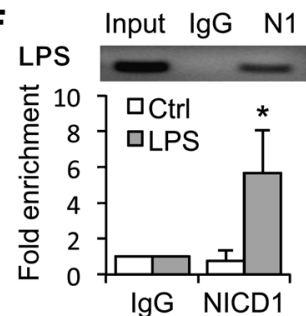

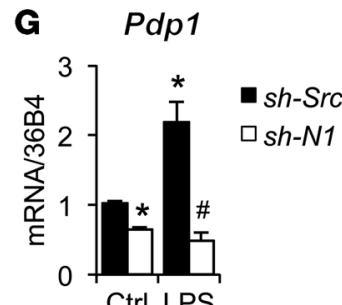

Figure 3. NOTCH reprograms M1 Macs to glucose mitochondrial oxidation through upregulation of PDP1. (A) Increased glucose uptake and lactate production by M1 Hmacs is attenuated with DAPT $(n=6) .{ }^{*} P<0.001,{ }^{*} P<0.05,1$-way ANOVA. (B) Primary HMacs were cultured with $99.9 \%$ [U-13 $C_{6}$ ]-glucose $(1 \mathrm{~g} / \mathrm{l})$, with or without DAPT. Percentage glucose flux to the TCA cycle was determined by mass spectrometry $(n=6)$. ${ }^{*} P<0.001,{ }^{*} P<0.05,1$-way ANOVA (C) Seahorse analysis of OCR in HMacs. ATP synthase inhibitor oligomycin (Oligo), mitochondrial uncoupling agent FCCP, and ETC inhibitors antimycin and rotenone (AR) were given at indicated times $(n=5) .{ }^{*} P<0.05$ vs. control or OF+Alc+DAPT, $t$ test. (D) Immunoblot of PDP1, PDK, and total and phosphoPDH-E1 $\alpha$ (pSer293) in HMacs isolated from WT and Notch1 KO mice with OF+Alc feeding. Imagel quantification of the pPDH-E1 $\alpha / P D H-E 1 \alpha$ ratio is shown $(n=3) .{ }^{*} P<0.05, t$ test. (E) PDH activity in Raw 264.7 cells infected with lentiviral scrambled or Notch1 shRNA, treated with or without LPS. Values are relative activity to the control $(n=6)$. ${ }^{*} P<0.05$ vs. sh-Scr, ${ }^{\#} P<0.05$ vs. sh-Scr + LPS, 2-way ANOVA. (F) ChIP-qPCR for NICD1 (N1) at the CSL site of $P d p 1$ promoter in Raw 264.7 cells stimulated with or without LPS for 4 hours. Values are fold enrichments relative to IgG $(n=3) .{ }^{*} P<0.05$ vs. control NICD1, $t$ test. (C) Expression of $P d p 1$ in Raw 264.7 cells infected with scrambled or Notch1 shRNA, stimulated with or without LPS for 24 hours $(n=3)$.

${ }^{*} P<0.05$ vs. scrambled control, ${ }^{*} P<0.05$ vs. scrambled LPS, 2-way ANOVA.

to the same CSL site, and the enrichments were further induced by LI stimulation (Figure 2B). The Nos 2 promoter luciferase activity induced by LPS and hypoxia was attenuated with DAPT (Figure 2C). Notch1 silencing diminished Nos 2 promoter activity induced by scrambled lentiviral vector and LPS (Figure 2D), whereas overexpression of NICD1 increased the promoter activity (Figure 2E). These results suggest that direct activation of the Nos 2 promoter by NICD1 mediates NOTCH1-dependent Nos 2 transcription.

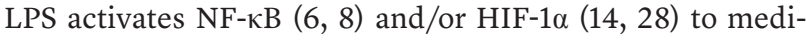
ate inflammatory responses of Macs. In Notch1 KO HMacs and in Raw 264.7 cells with Notch1 silencing, both basal and LPSinduced accumulation of nuclear p65 and HIF-1 $\alpha$ proteins was decreased (Figure 2F), suggesting their activation by LPS is partially dependent on NOTCH1. To understand the relative contribution of CSL, NF-KB, and HRE sites to the Nos 2 transcription, we performed site-directed mutagenesis to each site. The mutation on the CSL site increased the basal promoter activity but abrogated the promoter responsiveness to LPS stimulation and DAPT inhibition under both normoxia and hypoxia (Figure 2, G and H). This is consistent with the repressor role of CSL in transcrip- tional regulation (5) and suggests that NICD1 converts CSL to a coactivator complex at the Nos 2 promoter. The promoter activity was decreased with a NF- $\mathrm{kB}$ mutation under normoxia (Figure $2 \mathrm{G}$ ) and with a HRE mutation under hypoxia (Figure $2 \mathrm{H}$ ). In both $\mathrm{NF}-\kappa \mathrm{B}$ and HRE mutants, however, the Nos 2 promoter retained its responsiveness to LPS stimulation and DAPT suppression (Figure 2, G and H). Together, these results demonstrate that LPS-stimulated full Nos 2 transcriptional activity requires NICD1 binding to the proximal CSL site and the cooperative activities of adjacent NF- $\mathrm{\kappa B}$ and HRE sites.

Notch reprograms glucose metabolism to mitochondrial oxidation in M1 Macs. Enhanced glycolysis is seen in LPS-stimulated Macs (29) and dendritic cells (30) and in cancer cells overexpressing NICD1 (15). Indeed, M1 HMacs from the OF+Alc model exhibited increased glucose uptake and lactate production, depicting increased glycolysis, which are blocked by ex vivo DAPT treatment (Figure 3A). However, they also exhibited a $23.4 \%$ increase in glucose flux to the TCA cycle compared with the control cells, as determined by stable isotope $\left[\mathrm{U}-{ }^{13} \mathrm{C}_{6}\right]$-glucose flux analysis (Figure 3B). These M1 HMacs had increased basal and FCCP-induced oxy- 
A
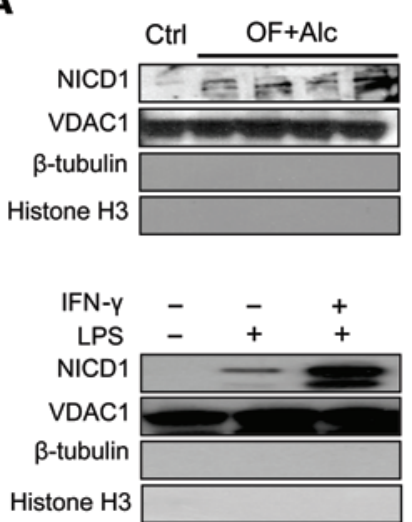

B
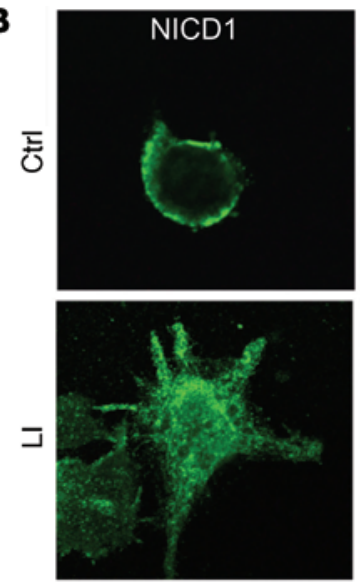
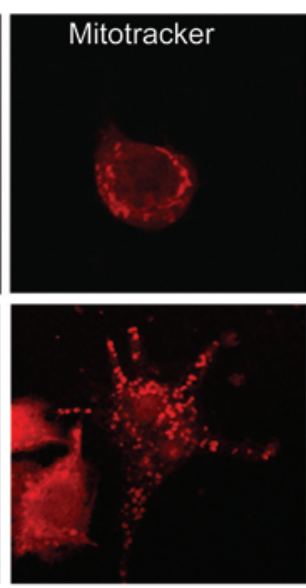
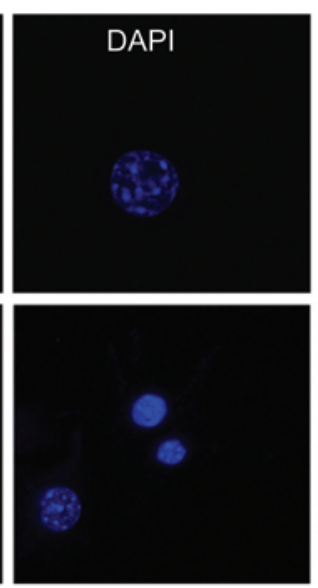
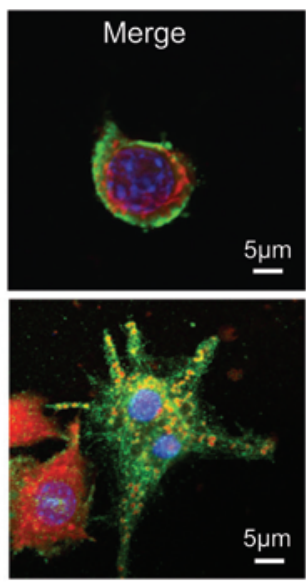

C

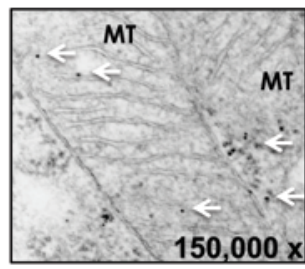

D

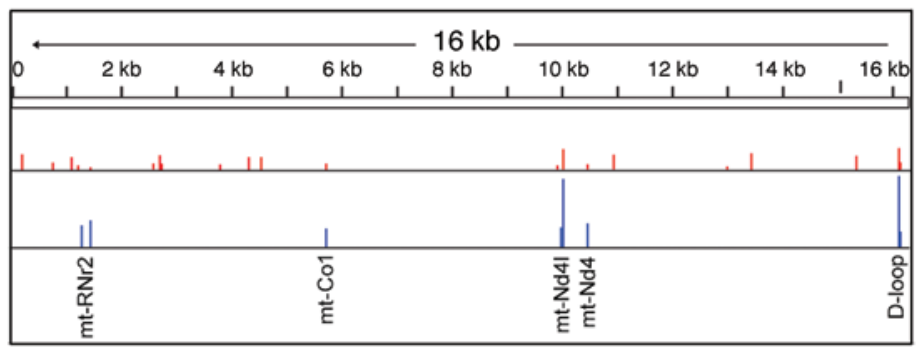

E
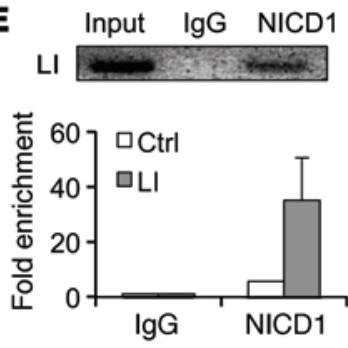

Figure 4. NOTCH regulates mtDNA transcription. (A) Immunoblot of NICD1 of mitochondrial proteins from control or OF+Alc HMacs and Raw 264.7 cells treated with or without LPS or IFN- $\gamma$ for 24 hours. VDAC1 served as a loading control, and the absence of $\beta$-tubulin or histone H3 validates the purity of mitochondrial proteins. (B) Fluorescent confocal microscopy shows the colocalization of NICD1 (green) and MitoTracker (red) in Raw 264.7 cells treated with LPS plus LI for 24 hours. Results are representative of 5 different experiments. Scale bars: $5 \mu$ m. (C) Electron microscopy image (original magnification, $\times 150,000)$ of immunogold-NICD1 in mitochondria (MT) of Raw 264.7 cells stimulated with LPS for 24 hours, as indicated by arrows. Results are representative of 3 different experiments. (D) ChIP-seq analysis on mtDNA from Raw 264.7 cells stimulated with LPS for 24 hours. Integrative Genomics Viewer genome browser tracks show the levels of NOTCH1 ChIP sample (blue) over input (red). Different genomic coordinates and genome window size for ChrM (chr17:23,695,78623,710,730; $15 \mathrm{~kb}$ ) are shown along with mm9 RefSeq data. (E) Mitochondrial ChIP-qPCR with primers specific for the D-loop region (15,752-15,903 bp) of the mitochondrial genome in Raw 264.7 cells stimulated with LPS for 24 hours. Values are fold enrichments relative to control or LPS IgC and represent 1 pooled mtDNA sample from $10 \times 10 \mathrm{~cm}$ plates of control cells and 2 pooled mtDNA samples from $10 \times 10 \mathrm{~cm}$ plates per each LPS-treated cell.

gen consumption rates (OCRs) (Figure 3C), suggesting enhanced oxidative phosphorylation (OXPHOS) and spared respiratory capacity $(31,32)$. These metabolic changes were all attenuated with DAPT (Figure 3, B and C). The NOTCH-dependent glucose metabolism and OCR in M1 HMacs were confirmed in M1 Raw 264.7 cells (Supplemental Figure 2, A and B). Collectively, these results demonstrate that NOTCH1 activation, which is essential for Mac M1 activation, promotes glucose flux to OXPHOS, while concurrently stimulating glycolysis.

Notch activates $P D H$ through upregulation of $P D H$ phosphatase. $\mathrm{PDH}$ (E1), composed of a heterotetramer of $\alpha$ and $\beta$ subunits, is the key component of PDH complex that shunts glucose flux to the TCA cycle. The PDH activity is suppressed via phosphorylation of the PDH-E1 $\alpha$ subunit at 3 serine sites, including Ser293, by PDH kinase (PDK) and conversely enhanced via dephosphorylation of these sites by PDH phosphatase (PDP) catalytic subunit 1 (PDP1) $(33,34)$. In HMacs isolated from Notch1 KO mice with OF+Alc feeding, PDP1 proteins were significantly decreased, while PDK proteins were unaffected, compared with those isolated from WT mice (Figure 3D). Both pPDH-E1 $\alpha$ (pSer293) and PDH-E1 $\alpha$ proteins were decreased in the Notch1 KO HMacs, and densitometric analysis revealed an increased ratio of pPDH-E1 $\alpha / \mathrm{PDH}-\mathrm{E} 1 \alpha$ (Fig- ure 3D), suggesting decreased PDH activity. The NOTCH1-dependent changes in protein levels of PDP1, pPDH-E1 $\alpha$, and PDH-E1 $\alpha$ as well as the ratio of $\mathrm{pPDH}-\mathrm{E} 1 \alpha / \mathrm{PDH}-\mathrm{E} 1 \alpha$ were also confirmed in Raw 264.7 cells with Notch1 silencing (Supplemental Figure 2C). As expected from these results, Notch1 silencing reduced LPSinduced PDH activity in M1 Macs (Figure 3E).

To understand the molecular mechanism for the NOTCH1dependent upregulation of PDP1, we screened the mouse Pdp1 gene and identified the putative CSL site (-522 GCGGGAA -516) within its proximal promoter. ChIP-qPCR revealed increased enrichment of NICD1 on this site in M1 Raw 264.7 cells (Figure 3 F). NOTCH1 silencing diminished the basal and LPS-induced Pdp1 mRNA levels (Figure 3G) but had no effects on expression of Pdha1 (Supplemental Figure 2D), which encodes PDH-E1 $\alpha$. Collectively, these results suggest that NOTCH1 increases PDH activity via its transcriptional activation of PDP1 to reduce the relative abundance of $\mathrm{PPDH}-\mathrm{E} 1 \alpha$, resulting in increased $\mathrm{PDH}-$ E1 $\alpha$ activity and glucose flux to the TCA cycle for OXPHOS in M1 Macs. Furthermore, the NOTCH1-dependent expression of the total PDH-E1 $\alpha$ protein that we have observed may also be related to PDP1 regulation, because the stability of PDH-E1 $\alpha$ is negatively correlated with its phosphorylation levels (35). 
A

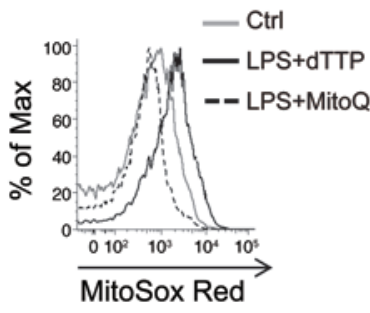

$\mathbf{B}$

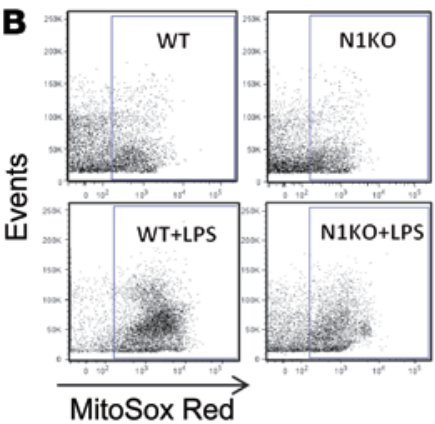

D
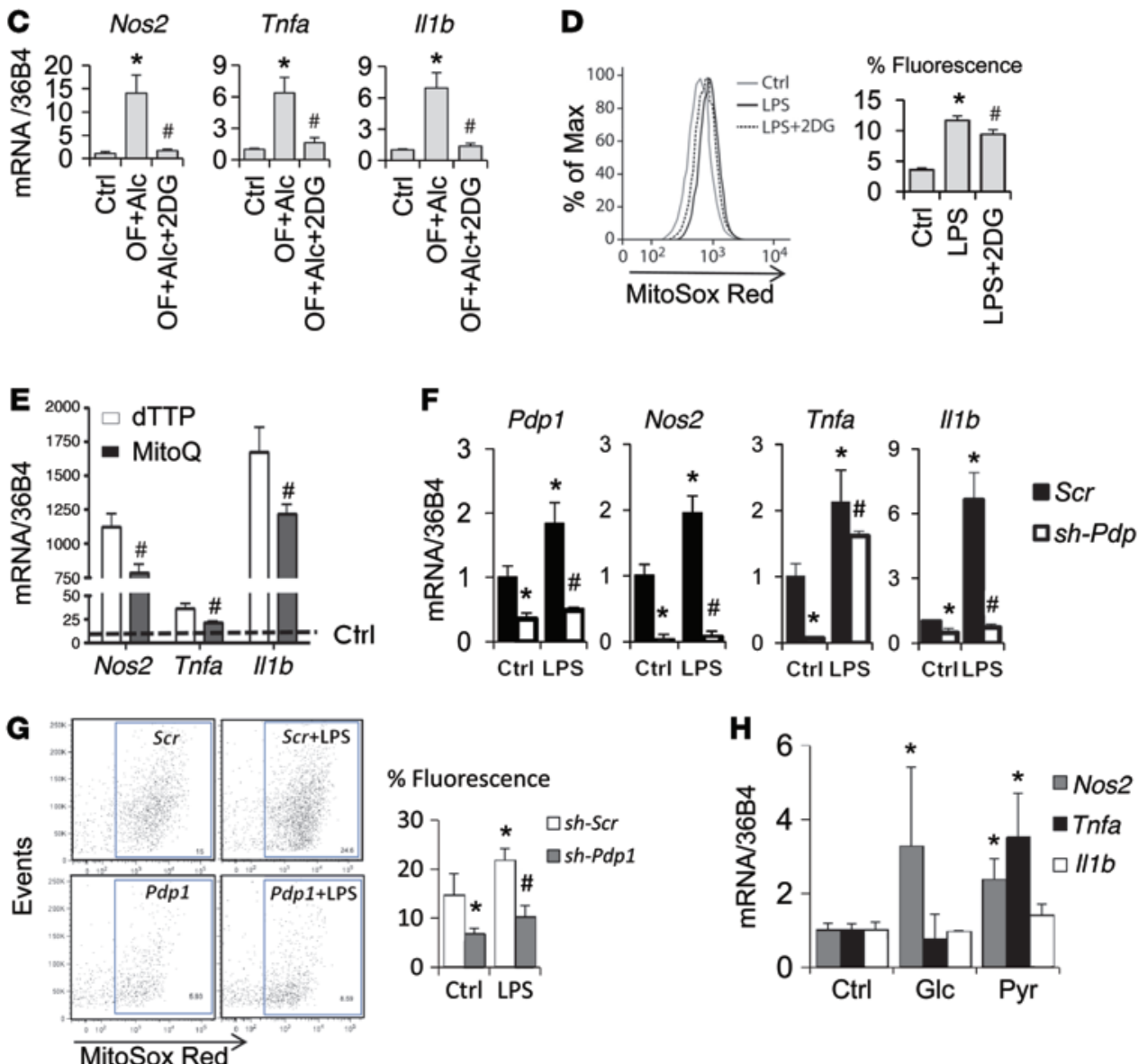

Figure 5. NOTCH-dependent glucose oxidation and mtROS augment M1 activation. (A) FACS analysis of mtROS using MitoSox Red in WT primary HMacs pretreated with or without dTTP or MitoQ for 1 hour followed by LPS for 24 hours. dTTP served as a pharmacologic control for MitoQ $(n=4-6)$. ${ }^{*} P<0.05$ vs. control, ${ }^{\#} P<0.05$ vs. LPS plus dTTP, 1-way ANOVA. (B) FACS analysis of mtROS using MitoSox Red in WT or Notch1 KO HMacs treated with or without LPS for 24 hours $(n=6-8) .{ }^{*} P<0.05$ vs. WT, ${ }^{\#} P<0.05$ vs. WT+LPS, 1-way ANOVA. (C) 2-DG reduces the expression of M1 genes in HMacs from $\mathrm{OF}+$ Alc mice $(n=3) .{ }^{*} P<0.05$ vs. control, ${ }^{\#} P<0.05$ vs. OF+Alc, 1-way ANOVA. (D) 2-DG attenuates $\mathrm{mtROS}$ in LPS-induced M1 Raw 264.7 cells $(n=3) .{ }^{*} P<0.05$ vs. control, $\# P<0.05$ vs. LPS, 1-way ANOVA. (E) MitoQ suppresses the expression of $\mathrm{M} 1$ genes in M1 Raw 264.7 cells treated with LI for 4 hours $(n=3)$. The dashed line indicates the mRNA levels of control cells, which are set to $1 .{ }^{\#} P<0.05$ vs. LI+dTTP, $t$ test. (F and $\mathbf{G})$ Pdp1 silencing abrogates the expression of (F) M1 genes or (G) mtROS in Raw 264.7 cells infected with scrambled or Pdp1 shRNA with or without LPS treatment $(n=4-6) .{ }^{*} P<0.01$ vs. Scr control, ${ }^{P} P<0.01$ vs. Scr LPS 2-way ANOVA. (H) Expression of M1 genes in WT HMacs cultured in glucose/ pyruvate-free medium (control) or supplemented with either glucose $(5.5 \mathrm{mM})$ or pyruvate $(10 \mathrm{mM})(n=6)$. ${ }^{*} P<0.05$ vs. control, $t$ test.
Notch regulates mitochondrial DNA transcription. Mitochondrial OXPHOS produces ATP through sequential reduction of respired oxygen via the electron transport chain (ETC) complexes, which are composed of subunits encoded by both nuclear genes and mitochondrial DNA (mtDNA). In M1 Raw 264.7 cells, our genome-wide ChIP-seq analysis also showed NICD1 enrichments at nuclear genes encoding ETC components (Atp5k, Ndufs7, $N d u f s 8, U q c r c 2)$ and proteins involved in ETC assembly (Ecsit), mtDNA replication (Dna2), and mitochondrial protein synthesis (Hemk1, Mrpls, Dars2) (Supplemental Table 1). Supplemental Figure 3A shows an example of the NICD1 enrichment at the ETC complex I subunit $N d u f s 7$. We screened the gene expression of ETC components in both M1 HMacs and M1 Raw 264.7 cells using TaqMan array and showed NOTCH-dependent expression of mtDNA-encoded NADH dehydrogenase, cytochrome b, cytochrome c oxidase, and ATP synthases (Supplemental Table 2).
These changes in mtDNA expression are unlikely to have resulted from changes in mitochondrial size and numbers (Supplemental Figure 3, B and C). In testing the role of NICD1 in mtDNA transcription, we revealed that NICD1 translocates to mitochondria in M1 Macs by immunoblotting of mitochondrial proteins (Figure 4A), by fluorescent confocal microscopy demonstrating NICD1-mitochondria colocalization (Figure 4B), and by electron microscopy showing immunogold-NICD1 in mitochondria (Figure 4C and Supplemental Figure 3D). Further, ChIP-seq performed with mtDNA fragments prepared from isolated mitochondria showed NICD1 enrichments at several sites within the mitochondrial genome $(P<0.05)$, including the noncoding region of the displacement loop (D-loop) (Figure 4D), which contains promoters responsible for mtDNA transcription (36). The recruitment of NICD1 to the D-loop was confirmed by ChIP-qPCR using isolated mtDNA fragments (Figure 4E). These results suggest the 


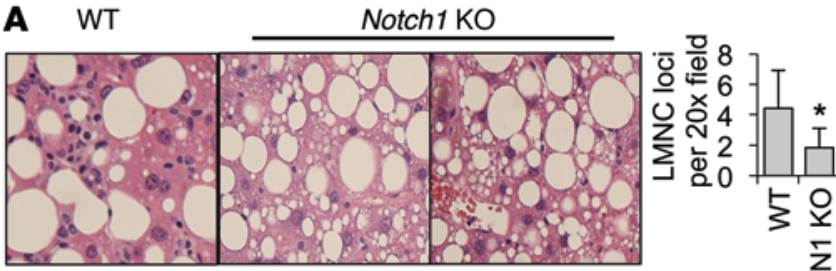

C

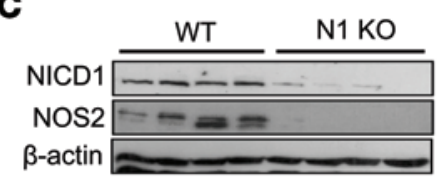

D

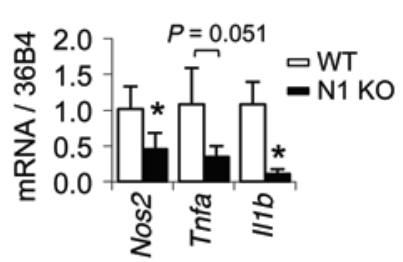

B

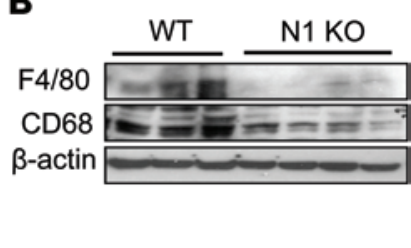

E

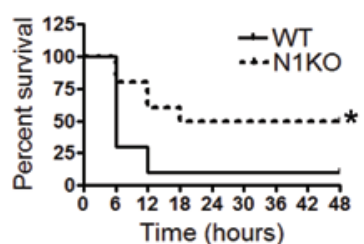

$\boldsymbol{F}$
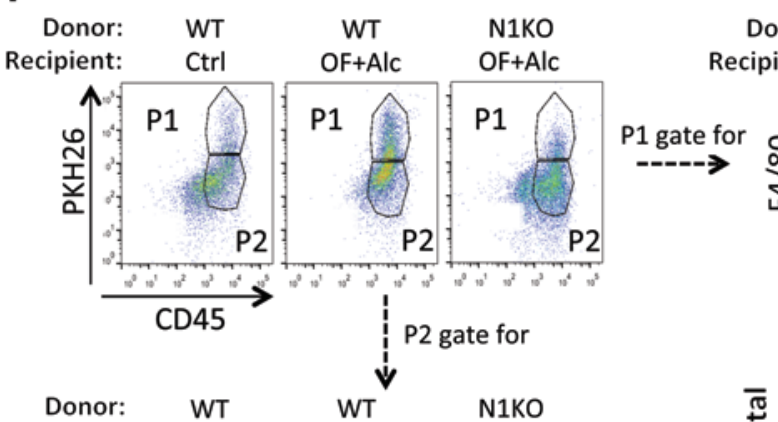

Donor: WT

WT
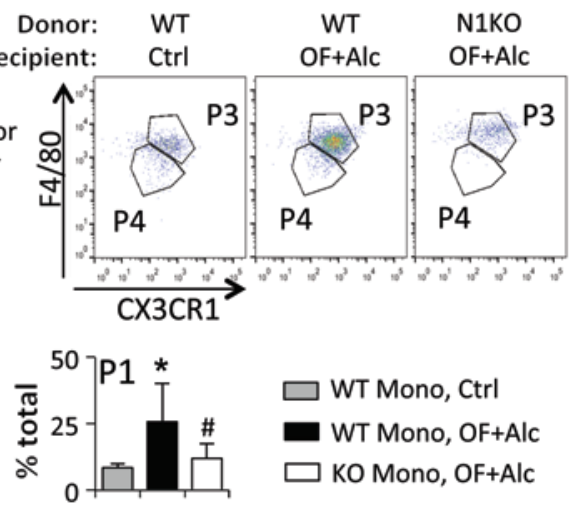

Recipient:

Ctrl OF+Alc OF+Alc
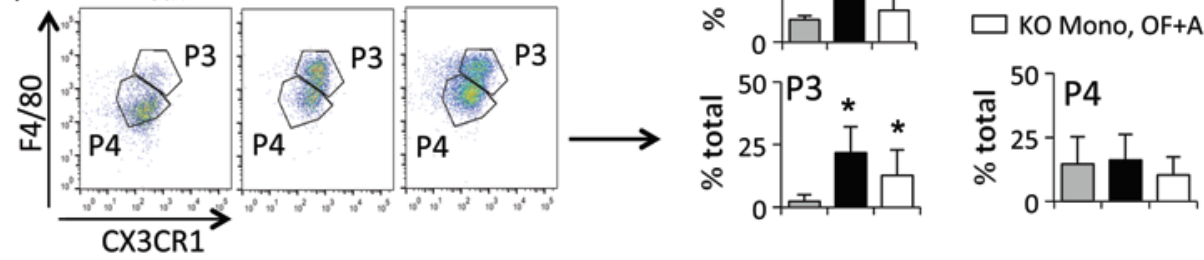

G

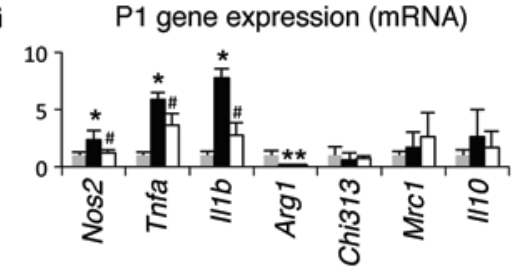

P4 gene expression (mRNA)

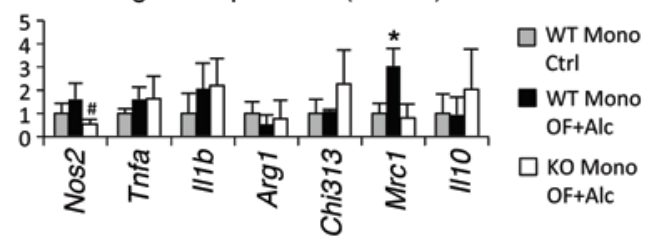

Figure 6. Inhibition of NOTCH pathway in vivo attenuates HMac M1 activation and liver inflammation. (A) Representative liver histology (H\&E; original magnification, $\times 200$ ) of WT and Notch1 KO mice with $\mathrm{OF}+$ Alc feeding, with average loci of liver mononuclear cells (LMNCs) per $\times 200$ optical field $(n=6) .{ }^{*} P<0.05$, $t$ test. (B and C) Immunoblot of (B) $\mathrm{CD} 68$ and $F 4 / 80$ in the livers and (C) NICD1 and NOS2 in HMacs of WT and myeloid Notch $1 \mathrm{KO}$ mice with $\mathrm{OF}+$ Alc feeding. (D) Expression of M1 genes in HMacs from the WT and Notch1 KO mice with OF+Alc feeding $(n=6) .{ }^{*} P<0.05$ vs. WT, $t$ test. (E) Survival rate of mice injected intravenously with galactosamine (350 $\mathrm{mg} / \mathrm{kg})$ and LPS $(4 \mu \mathrm{g} / \mathrm{kg})$ in $200 \mu \mathrm{l}$ PBS $\left(n=10\right.$ per group). ${ }^{*} P<0.01$, log-rank test. (F) FACS analysis of HMacs isolated from WT mice receiving WT or Notch1 $\mathrm{KO}$ donor monocytes. After gating on CD45, HMacs were separated into $\mathrm{PKH}^{2} 6^{+}$ $\mathrm{P} 1$ and $\mathrm{PKH} 26^{-} \mathrm{P} 2$ populations, which were further separated into $\mathrm{P} 3$ and $\mathrm{P} 4$ populations using Mac markers F4/80 and CX3CR1. The percentage of $P 1, P 3$, and $P 4$ cells of total HMacs are presented in bar graphs $(n=6-8)$. Mono, monocytes. ${ }^{*} P<0.05$ vs. WT donor and control recipient mice, ${ }^{\#} P<0.05$ vs. WT donor and $\mathrm{OF}+$ Alc recipient mice, 1-way ANOVA. (C) Expression of M1 vs. $M 2$ genes in infiltrating $P 1$ donor monocytes/Macs and in resident P4 recipient Kupffer cells ( $n=6-8)$. ${ }^{*} P<0.05$ vs. WT donor and control recipient mice, ${ }^{\#} P<0.05$ vs. WT donor and $\mathrm{OF}+$ Alc recipient mice, 1-way ANOVA. novel positive regulatory role of the NOTCH1 pathway in mtDNA transcription and mitochondrial respiration in M1 Macs.

Mitochondrial ROS-mediated M1 gene expression depends on Notch1 and glucose oxidation. OXPHOS via ETC is a major source of mtROS (37). Increased mtROS is seen in M1 Macs and implicated in innate immune function and cytokine expression $(38,39)$. Indeed, mtROS was increased in LPS-induced M1 HMacs (Figure 5A) and M1 Raw 264.7 cells (Supplemental Figure 4A) and attenuated by pretreatment with mitochondrial-specific superoxide scavenger MitoQ (37). More importantly, LPS-induced mtROS was abrogated in NOTCH1 KO HMacs (Figure 5B) and in Raw 264.7 cells with NOTCH1 silencing (Supplemental Figure 4B) or DAPT pretreatment (Supplemental Figure 4C), demonstrating Notch1-dependent mtROS generation in M1 Macs.

The observed NOTCH1-dependent increases in glucose oxidation, mitochondrial respiration, and mtROS in M1 Macs suggest the causal relationships among them. To address this question, we first used the hexokinase inhibitor 2-deoxyglucose (2-DG) to examine its effects on mtROS generation and M1 gene expression. In M1 HMacs, 2-DG abrogated the increased mRNA of M1 genes (Figure $5 \mathrm{C}$ ) and production of TNF- $\alpha$, IL-1 $\beta$, IL-12, and MIP- $1 \alpha$ cytokines (Supplemental Figure 4D). Etomoxir (ETO), an inhibitor of fatty acid oxidation, had no effect on this cytokine production (Supplemental Figure 4D). These results with 2-DG and ETO were confirmed in M1 Raw 264.7 cells (Supplemental Figure 4E). In these cells, 2-DG also reduced mtROS (Figure 5D) and MitoQ attenuated M1 gene expression (Figure 5E), suggesting that 2-DG's inhibitory effect on M1 genes is mediated via reduced mtROS.

To validate the notion that M1 gene expression is dependent on glucose influx to mitochondrial TCA facilitated by NOTCH1mediated Pdp1 upregulation, we knocked down Pdp1 in Raw 2647 cells. Indeed, this manipulation diminished basal and LPS- 


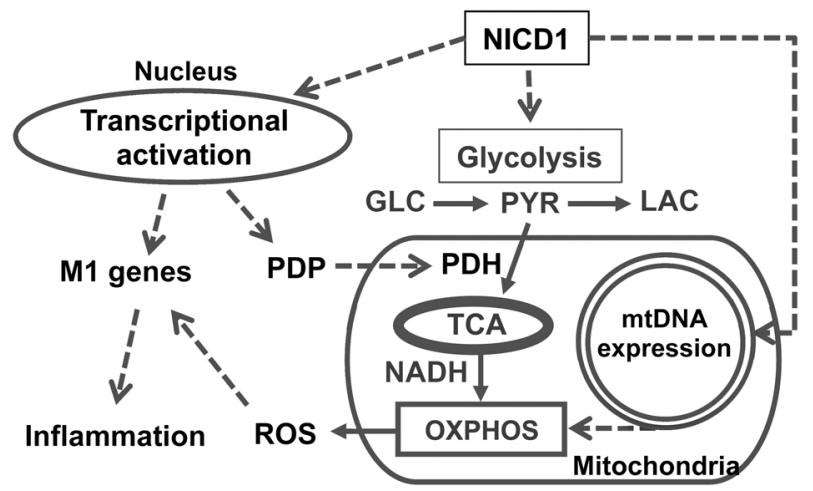

induced M1 gene expression (Figure 5F) and mtROS (Figure 5G). In WT HMacs cultured in glucose/pyruvate-free media, supplementation with pyruvate increased M1 gene expression (Figure $5 \mathrm{H})$. Collectively, these results support the critical role of a glucose/pyruvate/mitochondria metabolic axis for mtROS generation and Mac M1 activation.

Notch inhibition in myeloid cells attenuates HMacs M1 activation and liver inflammation. Our ex vivo and in vitro studies described above demonstrate NOTCH1-dependent pathways for Mac M1 activation. To evaluate in vivo the causal role of NOTCH1 in M1 activation and inflammation in ASH, we inhibited the NOTCH pathway in OF+Alc mice using two approaches. First, we administrated DAPT (10 mg/kg, i.p.) (23) to the OF+Alc mice, and this treatment had minimal effect on portal LPS levels but significantly decreased plasma ALT - the biochemical index of liver injury (Supplemental Figure 5A). DAPT did not prevent the histological grading of steatosis but attenuated mononuclear cell infiltration (Supplemental Figure 5B) and reduced NICD1, NOS2, and F4/80 in the liver (Supplemental Figure 5C). To offset the global and off-target effects of DAPT and validate that the above findings were due specifically to NOTCH1 inhibition in myeloid cells, we fed myeloid Notch1 KO mice the OF+Alc diet. Like DAPT, Notch1 KO did not prevent steatosis but reduced mononuclear cell infiltration (Figure 6A) and Mac marker proteins CD68 and F4/80 in the liver (Figure 6B). In isolated HMacs, Notch1 KO downregulated NOS2 protein (Figure 6C) and M1 genes (Figure 6D) but not M2 genes (Supplemental Figure 5D). These results demonstrate that repressed HMac M1 activation secondary to NOTCH1 deficiency does not enhance the M2 phenotype. Interestingly, myeloid Notch1 KO also decreased liver ROS by $40 \%$ (Supplemental Figure 5E), indicating the contribution of NOTCH1-dependent M1 activation to hepatic oxidative stress in ASH. Last, we extended our studies to test the protective role of NOTCH1 deficiency in liver injury caused by excessive HMac M1 activation, using the galactosamine-sensitized, LPS-induced fulminant hepatitis model, which results in high lethality (40). As shown in Figure 6E, the 48-hour mortality rate of $90 \%$ in the WT mice was reduced to $50 \%$ in myeloid Notch1 KO mice, demonstrating the causal role of NOTCH1 in M1 HMac-dependent lethality in the model. Taken together, these results demonstrate the in vivo role of the NOTCH1 pathway in Mac M1 activation as what we believe to be a novel molecular mechanism of liver inflammation and injury.
Figure 7. NOTCH activation reprograms mitochondrial metabolism to augment Mac M1 gene expression. NOTCH1 activation results in liberation of NICD1, which translocates into the nucleus to activate transcription of M1 genes, such as Nos2 and the metabolic gene Pdp1. NOTCH1 activation enhances glycolysis and concurrent glucose flux to the TCA cycle through upregulation of $P d p 1$ and subsequent PDH activity. NICD1 also translocates to the mitochondrial and promotes mtDNA transcription in M1 Macs. The increased mtDNA expression and glucose oxidation lead to enhanced OXPHOS and consequent mtROS, which in turn augments expression of M1 genes. GLC, glucose; PYR, pyruvate; LAC, lactate.

The amelioration of hepatic inflammation seen in Notch1 KO mice may be a result of reduced migration of blood monocytes into the liver for subsequent generation of M1 HMacs or suppressed M1 activation of preexisting resident Kupffer cells. To clarify this issue, we purified blood monocytes from Notch1 KO and WT mice, labeled them with PKH26 fluorescent dye, and intravenously injected them into WT recipient mice subjected to OF+Alc feeding to track the migration of the donor monocytes into the liver (41). Migration of PKH26-labeled WT monocytes, but not Notch 1 KO monocytes, to the livers of $\mathrm{OF}+\mathrm{Alc}$ recipient mice was increased as compared with that of the controls (Supplemental Figure 5F). We isolated HMacs from the recipient mice and separated them into $\mathrm{CD} 45^{+} \mathrm{PKH} 26^{+}$donor (P1) monocytes and unlabeled CD45 ${ }^{+} \mathrm{PKH} 26^{-}$recipient (P2) monocytes/Macs (Figure 6F). The P1 and P2 populations were further separated into $\mathrm{F} 4 / 80^{+} \mathrm{CX} 3 \mathrm{CR} 1^{\text {hi }}(\mathrm{P} 3)$ and $\mathrm{F} 4 / 80^{+} \mathrm{CX} 3 \mathrm{CR} 1^{\text {lo }}(\mathrm{P} 4)$ populations, respectively (Figure 6F), which clearly demonstrate that $\mathrm{P3}$ represents infiltrating monocytes/Macs and $\mathrm{P} 4$ represents resident Kupffer cells (42). As shown in Figure 6F, FACS analysis shows a 3- to 5 -fold increase in migration of both WT donor P1 and recipient P3 monocytes/Macs to the livers of OF+Alc mice compared with that in the controls, while migration of Notch1 KO donor P1 monocytes/ Macs was comparable to that of the controls, and transplantation of Notch1 KO donor P1 cells had minimal effect on the migration of recipient P3 monocytes/Macs. Interestingly, neither OF+Alc feeding nor transplantation of Notch1 $\mathrm{KO}$ monocytes had a significant impact on the Kupffer cell population (Figure 6F). These results show that NOTCH1 is required for monocyte migration into the liver undergoing ASH. Further, the expression of M1 genes was upregulated in the OF+Alc mice receiving WT donor monocytes but significantly decreased in those with the Notch1 KO donor monocyte transplantation. The expression of M2 genes was not significantly different (Figure 6G). Expression of both M1 and M2 genes in resident Kupffer cells was not significantly affected by OF+Alc feeding and by monocyte transplantation (Figure 6G). Collectively, these results highlight the importance of NOTCH-dependent migration of monocytes and subsequent M1 differentiation in determining the extent of HMac expansion and inflammation in the livers of $\mathrm{OF}+$ Alc mice.

\section{Discussion}

The NOTCH pathway is conserved across species and promotes cell fate specification and differentiation of various cell types, such as skin (43) and biliary (44) epithelial cells, cardiac (45) and skeletal (46) myocytes, neuronal cells (47), and T cells (48). Based on 
the findings from the present study, M1 Mac differentiation may be added to this list of NOTCH-mediated cell fate regulation. Most notably, our study demonstrates the ability of the NOTCH pathway to stimulate glucose oxidation, OXPHOS, and mtROS generation and to couple this metabolic shift with NOTCH-dependent transcriptional activation of M1 genes to augment M1 activation. These metabolic effects are associated with NICD1 recruitment to the regulatory regions of both nuclear and mitochondrial genes that control glucose-pyruvate entry to the TCA cycle and mitochondrial respiration and with NOTCH-dependent upregulation of such genes, suggesting the role of NICD1-mediated transcriptional regulation in stimulating mitochondrial metabolism in M1 Macs (Figure 7). Further, our monocyte-tracking studies demonstrate that the NOTCH1 pathway is essential for migration of blood monocytes into the liver and subsequent M1 differentiation.

In our study, the Nos 2 gene was studied as a prototypic M1 gene, which plays a key role in nitrosative tissue damage in inflammation. The association of Nos 2 expression with metabolic switch in M1 Macs and Arg1 expression with metabolic switch in M2 Macs has been discussed previously (49); however, the underlying mechanisms for such association were not clear. The roles of NF- $\kappa \mathrm{B}(50)$ and HIF- $1 \alpha(14,51)$ in Mac M1 activation and Nos 2 expression are well-established. Cooperative interactions of the NOTCH pathway with these transcription factors have also been reported. For instance, the NOTCH pathway contributes to LPSstimulated and NF- $\kappa \mathrm{B}-$ mediated Mac M1 activation (6, 8). Hypoxia-induced HIF- $1 \alpha$ causes inflammation and Nos 2 upregulation $(14,51)$, and NOTCH and HIF-1 $\alpha$ interact to regulate cell differentiation and survival $(10,52)$. NOTCH activation upregulates IRF8 and promotes TLR-induced inflammatory polarization of Macs (53). In our study, NOTCH1 silencing attenuated both basal and LPS-induced nuclear accumulation of p 65 and HIF-1 $\alpha$, suggesting that activation of NF- $\kappa \mathrm{B}$ and HIF- $1 \alpha$ is at least partially dependent on NOTCH1. NICD1 converts the CSL corepressor complex to the NICD-CSL transcriptional activator, and such a derepression mechanism (54) appears required for NF- $\kappa$ B and/or HIF- $1 \alpha$ to fully activate Nos 2 transcription. As these 3 cis elements reside within a 158-bp span on the proximal promoter, their cooperative interactions can be easily assumed to be as proposed for NICD and NF- $\kappa \mathrm{B}$ $(8,55)$ or HIF-1 $\alpha(56)$. As NICD1 is enriched at other M1 genes and these genes are coordinately repressed by NOTCH inhibition, similar NICD-mediated cross-regulation of the other transcription factors may exist for a broader range of inflammatory genes.

Our study has identified Pdp1 as a target of the $\mathrm{NOTCH}$ pathway, which is at least in part responsible for increased mitochondrial glucose oxidation, mtROS, and M1 gene expression via reduced relative abundance of $\mathrm{pPDH}-\mathrm{E} 1 \alpha$ and increased $\mathrm{PDH}$ activity. Inhibition of $\mathrm{PDH}-\mathrm{E} 1 \alpha$ phosphorylation by dichloroacetate, a PDK inhibitor, decreases the E1 $\alpha$ degradation and maximizes the PDH activity (35). In contrast, phosphorylation of $\mathrm{PDH}-\mathrm{E} 1 \beta$ at tyrosine residues is associated with enhanced E1 $\beta$ degradation via ubiquitination and decreased PDH activity (57). Indeed, the most notable change in M1 Macs is NOTCH-dependent expression of the PDH-E1 $\alpha$ protein. In fact, the increased relative abundance of $\mathrm{PPDH}-\mathrm{E} 1 \alpha$ (pSer293) over $\mathrm{PDH}-\mathrm{E} 1 \alpha$ by Notch1 $\mathrm{KO}$ and silencing is largely attributable to depletion of total PDH-E1 $\alpha$ (Figure 3D and Supplemental Figure 2C). As PDH sub- unit mRNA expressions are not regulated, NOTCH1 must render translational or posttranslational control of $\mathrm{PDH}-\mathrm{E} 1 \alpha$ protein turnover through an unknown mechanism.

Activated phagocytes are generally thought to be glycolytic cells (58). The glycolysis in M1 Macs in our study is indeed increased, as evident by enhanced lactate production. ATP generation through glycolysis is 19 -fold less efficient than that by glucose oxidation. Although the rate of ATP production by glycolysis can be approximately 100 times faster than the rate of oxidation (59), we and others $(30,60)$ only observed a 2 -fold increase in glucose utilization in M1 Macs. Thus, the high velocity of the glycolysis cannot adequately support required ATP, supporting the notion that metabolic shift to glycolysis is energetically unfavorable for the phagocytic activity (61). On the other hand, we observed a $23.4 \%$ increase in glucose flux to the TCA cycle (Figure 3B), which will yield 4.2-fold more ATP based on the presumption that $2 \mathrm{ATP}$ is generated through glycolysis and $36 \mathrm{ATP}$ is generated through oxidation per glucose molecule. In fact, during phagocytosis Macs rely partly on glucose oxidation and OXPHOS, whereas neutrophils and monocytes may solely depend on glycolysis as a source of metabolic energy (58). Using ${ }^{14} \mathrm{C}$-labeled glucose, Karnovsky and colleagues have shown that conversion of $\left[6-{ }^{14} \mathrm{C}\right]-$ glucose to ${ }^{14} \mathrm{CO}_{2}$, the index for glucose oxidation in the TCA cycle, is about 4-fold higher in thioglycollate-elicited peritoneal Macs during phagocytosis than in resting Macs (62). Similarly, peritoneal Macs from mice challenged with Listeria monocytogenes exhibit increased glucose oxidation (63). Collectively, these results support enhanced glucose oxidation by mitochondria as a key metabolic feature of M1 Macs. Our results not only validate this notion but also establish the causal link between mitochondrial glucose oxidation and OXPHOS with M1 activation via demonstration of mtROS-enhanced M1 gene expression.

Recent studies also suggest that glutamine metabolism is important in Mac inflammatory response (64). LPS-induced HIF$1 \alpha$ stabilization and M1 activation are dependent on the accumulation of TCA intermediate succinate derived from glutamine pathway, suggesting glutamine as an anerplerotic source for TCA activity required for HIF- $1 \alpha$ and M1 activation (28). Interestingly, this glutamine-dependent effect is abrogated by 2-DG, demonstrating the requirement of glucose metabolism for this mechanism to function. This is due likely to the fact that the 2-carbon metabolite acetyl-CoA produced by $\mathrm{PDH}$ via glucose-pyruvate flux is critical for the TCA activity, and without it the glutamine pathway alone is not sufficient to drive the TCA cycle for succinate generation. Thus, the succinate-HIF- $1 \alpha$ link may be coupled with a NOTCH-dependent increase in glucose flux to TCA via enhanced PDH activity, as demonstrated by our study.

Our observation of NICD1 localization within mtDNA provides what we believe to be the novel molecular basis for the increase in mitochondrial respiration and mtROS. Mitochondria localization of NICD has been reported previously (65). However, the mechanism for NICD1 translocation to mitochondria remains unknown. Nuclear DNA-encoded mitochondrial proteins contain mitochondrial targeting signals (MTSs) with an amphipathic helix structure at their N-terminus. NICD1 has a putative MTS within the 20 amino acids at its N-terminus, as predicated by a bioinformatics program PSORT (ref. 66, 
http://psort.hgc.jp) and as suggested by a previous study (67) Mitochondrial intermediate peptidase may cleave the N-terminal MTS of NICD1 and release a truncated NICD1 in mitochondria in vitro (67). Although ChIP-seq and ChIP-qPCR showed NICD1 recruitment to the mtDNA D-loop, we could not identify a consensus CSL site [G(t)TGGGAAA(c); lowercase letters in parentheses represent letters that can be substituted for the preceding capital letters] in this region. In a recent genome-wide analysis of NOTCH1/CSL binding in lymphoblastic leukemia cells, only $36 \%$ of NICD1-binding events are shown to overlap with the CSL sites (68). In addition, Demehri et al. demonstrate the existence of CSL-independent NOTCH-mediated regulation in keratinocytes and epidermal differentiation (69). Thus, we speculate that the putative MTS on NICD1 guides its translocation to mitochondria, in which NICD1 binds to the D-loop and regulates mtDNA expression through interaction(s) with DNA-binding protein(s) other than CSL.

The mitochondrial ETC is the major source for cellular ROS, which is known to activate NF- $\mathrm{BB}$ and stabilize HIF-1 $\alpha$ (70-72), the two transcriptional factors we proposed to cooperate with NICD1 in regulation of Nos 2 expression. Although ROS generated by NADPH oxidase is considered a major defense mechanism against bacterial infection (73), evidence suggests that mtROS also contributes to bactericidal activity $(74,75)$. In LPS-stimulated Raw 264.7 cells, TLR signaling enhances the generation of mtROS through the TRAF6/ECSIT pathway to augment intravesicular bactericidal activity of M1 Macs (39). ECSIT is implicated in mitochondrial ETC complex I assembly (76) and identified as a NOTCH1 target by our genome-wide ChIP-seq analysis (Supplemental Table 1), suggesting a potential cross-interaction between the TRAF6/ECSIT pathway and the NOTCH pathway. Nos 2 gene upregulation and enhanced production of NO are known factors for antimicrobial actions of Macs (50). Excessive formation of $\mathrm{NO}$ may inhibit ETC activity through posttranslational modification of ETC component proteins (77). Yet, our data generated with Seahorse analysis show a significant increase in OCR after M1 Macs were treated with the uncoupler FCCP, indicating that these cells have higher spare respiratory capacity for survival. This may be related to the ability of NOTCH1 to support antiapoptotic activity and cell survival through AKT and the mitochondrial remodeling protein Mitofusin (65). In addition, NO may also enhance a cGMP-dependent mechanism in support of ATP generation (78). However, our morphometric results failed to support increased mitochondrial biogenesis in M1 HMacs.

In conclusion, using in vivo- and in vitro-activated M1 Macs, we demonstrate that NOTCH activation plays a key role in M1 gene expression and reprogramming of mitochondrial metabolism to augment M1 activation (Supplemental Figure 1). The NOTCH pathway links cell metabolism to the M1 fate of Macs via its ability to orchestrate coordinated stimulation of the two major components of OXPHOS, the TCA cycle and the ETC. The in vivo relevance of NOTCH-dependent Mac M1 activation is demonstrated by attenuated liver inflammation by pharmacologic or genetic inhibition of the NOTCH1 pathway in the mouse model of ASH as well as in mice with galactosamine/LPS-induced fulminant hepatitis. Using ex vivo monocyte labeling and in vivo tracking methods, we further demonstrate that hepatic inflammation in ASH was mediated by infiltration and M1 activation of peripheral blood monocytes facilitated by the NOTCH1 pathway. Our results imply a therapeutic role of NOTCH1 in M1 Mac-dependent inflammation.

\section{Methods}

Animals. Notch1-floxed mice (007181) and myeloid cell-specific LyzMCre mice (004781) from The Jackson Laboratory were crossed to generate the myeloid-specific Notch1 KO mice.

Intragastric feeding ASH mouse model. The WT and myeloid Notch1 KO mice were fed control, high-fat (40\% fat calories), alcohol (32 g/ $\mathrm{kg} / \mathrm{d}$ ), or combined $\mathrm{OF}+\mathrm{Alc}$ diet through intragastric tubing using the protocols described previously $(19,79)$. (Details of the model are described in the Supplemental Methods.) For global inhibition of the NOTCH pathway, the OF+Alc mice were administrated DAPT (10 $\mathrm{mg} / \mathrm{kg}$ in $5 \%$ alcohol plus $95 \%$ corn oil, i.p.) or a vehicle (5\% alcohol plus $95 \%$ corn oil), with a 3 -day-on and 4 -day-off schedule in the last 4 weeks of the 7-week feeding regimen (23).

Cell culture. The HMacs were isolated by The Southern California Research Center for ALPD and Cirrhosis nonparenchymal liver cell core using collagenase perfusion and digestion, followed by low-speed centrifugation $(50 \mathrm{~g})$ to remove parenchymal cells. The nonparenchymal cells were subjected to discontinuous gradient ultracentrifugation using OptiPrep (Axis-Shield PoC As.) to collect the HMacs fraction and cultured for 16 hours (for details, see the Supplemental Methods). The Abelson leukemia virus-transformed murine Mac Raw 264.7 cells were purchase from ATCC (TIB-71). In vitro activation of M1 Raw 264.7 cells was all carried out by treating the cells with LPS $(100 \mathrm{ng} / \mathrm{ml})$ with or without IFN- $\gamma(20 \mathrm{ng} / \mathrm{ml})$ for 4 or 24 hours, as specified in figure legends. NOTCH inhibition was achieved by pretreatment of the cells with DAPT ( $20 \mu \mathrm{M}, 16$ hours) unless otherwise specified or by lentiviral sh-Notch1 silencing. For experiments under hypoxia, both HMacs and Raw 264.7 cells were cultured in a incubator and shuttle glove box (BioSpherix) set at $2 \% \mathrm{O}_{2}$. For inhibition of glycolysis or fatty acid oxidation, the cells were pretreated with 2-DG $(5.5 \mathrm{mM}, 1$ hour $)$ and ETO ( $500 \mu \mathrm{M}, 1$ hour), respectively, before other treatments were initiated.

Mitochondrial fractionation and purification. Mitochondrial fractionation was carried out using 2-step Percoll gradient centrifugation to ensure purity (for details, see the Supplemental Methods).

ChIP and ChIP-seq. Detailed procedures and bioinformatics analysis can be found in the Supplemental Methods. The ChIP-seq data have been deposited in the NCBI GEO database (accession GSE65151).

Other methods. Other experimental procedures, including materials, antibodies, primers, qRT-PCR, immunoblotting, monocytes purification, ex vivo labeling and in vivo tracking, galactosamine/LPS lethality test, isolation and culture of primary HMacs, ChIP and ChIP-seq, immunofluorescence and confocal microscopy, immunogold staining of NICD1 and electron microscopy, lentiviral shRNA silencing, morphology evaluation, cytokine measurement, site-directed mutagenesis and dual-luciferase reporter assay, Seahorse XF-24 metabolic flux analysis, stable isotope-based glucose flux analysis, and PDH activity assay, are described in detail in the Supplemental Methods.

Statistics. All numerical data are presented as mean \pm SD. Statistic significance was analyzed with 2-tailed distribution and 2-sample unequal variance Student's $t$ test, 1-way ANOVA followed by Newman-Keuls multiple comparison test, or 2-way ANOVA followed by Bonferroni post tests, as indicated in the figure legends. For survival studies, statistical analysis was determined by log-rank test using 
built-in Prism software (GraphPad). $P$ values of less than 0.05 were considered statistically significant.

Study approval. The animal experimental procedures were approved by the institutional animal care and use committee of the University of Southern California.

\section{Acknowledgments}

We thank Jianghong Wang and Raul Lazaro for their technical assistance. This study was supported by NIH grants P50AA011999 (to H. Tsukamoto), R24AA12885 (to H. Tsukamoto), K01AA020524 (to J. Xu), and DK048522-19 (pilot project grant to J. Xu) and by ABMRF/The Foundation for Alcohol Research (to J. Xu), the Medical Research Service of Department of Veterans Affairs grant 5IO BX001991-02F (to H. Tsukamoto), the pilot project from USC Research Center for Liver Diseases (to J. Xu), and Suntory Global Innovation Center Ltd. (to H. Tsukamoto).

Address correspondence to: Jun Xu, 1333 San Pablo Street, MMR 410, Los Angeles, California 90089-9141, USA. Phone: 323.442.3120; E-mail: junx@usc.edu.
1. Murray PJ, Wynn TA. Protective and pathogenic functions of macrophage subsets. Nat Rev Immunol. 2011;11(11):723-737.

2. Lacy-Hulbert A, Moore KJ. Designer macrophages: oxidative metabolism fuels inflammation repair. Cell Metab. 2006;4(1):7-8.

3. Agathocleous M, Harris WA. Metabolism in physiological cell proliferation and differentiation. Trends Cell Biol. 2013;23(10):484-492.

4. Artavanis-Tsakonas S, Rand MD, Lake RJ. Notch signaling: cell fate control and signal integration in development. Science. 1999;284(5415):770-776.

5. Mumm JS, Kopan R. Notch signaling: from the outside in. Dev Biol. 2000;228(2):151-165.

6. Palaga $T$, et al. Notch signaling is activated by TLR stimulation and regulates macrophage functions. Eur JImmunol. 2008;38(1):174-183.

7. Nilsson R, et al. Transcriptional network dynamics in macrophage activation. Genomics. 2006;88(2):133-142.

8. Monsalve E, et al. Notch1 upregulates LPS-induced macrophage activation by increasing NF- $\mathrm{kB}$ activity. Eur JImmunol. 2009;39(9):2556-2570.

9. Wang YC, et al. Notch signaling determines the $\mathrm{M} 1$ versus $\mathrm{M} 2$ polarization of macrophages in antitumor immune responses. Cancer Res. 2010;70(12):4840-4849.

10. Gustafsson MV, et al. Hypoxia requires notch signaling to maintain the undifferentiated cell state. Dev Cell. 2005;9(5):617-628.

11. Sahlgren C, Gustafsson MV, Jin S, Poellinger L, Lendahl U. Notch signaling mediates hypoxia-induced tumor cell migration and invasion. Proc Natl Acad Sci U S A. 2008;105(17):6392-6397.

12. Greijer AE, et al. Up-regulation of gene expression by hypoxia is mediated predominantly by hypoxia-inducible factor 1 (HIF-1). J Pathol. 2005;206(3):291-304.

13. Chandel NS, Trzyna WC, McClintock DS, Schumacker PT. Role of oxidants in NF-кB activation and TNF- $\alpha$ gene transcription induced by hypoxia and endotoxin. J Immunol. 2000;165(2):1013-1021.

14. Cramer T, et al. HIF-1 $\alpha$ is essential for myeloid cell-mediated inflammation. Cell. 2003;112(5):645-657.

15. Landor SK, et al. Hypo- and hyperactivated Notch signaling induce a glycolytic switch through distinct mechanisms. Proc Natl Acad Sci US A. 2011;108(46):18814-18819.

16. Wheeler MD. Endotoxin and Kupffer cell activation in alcoholic liver disease. Alcohol Res Health. 2003;27(4):300-306.

17. Baffy G. Kupffer cells in non-alcoholic fatty liver disease: the emerging view. J Hepatol. 2009;51(1):212-223.

18. Olefsky JM, Glass CK. Macrophages, inflammation, and insulin resistance. Annu Rev Physiol. 2010;72:219-246

19. Xu J, et al. Synergistic steatohepatitis by moderate obesity and alcohol in mice despite increased adiponectin and p-AMPK. J Hepatol. 2011;55(3):673-682.

20. Alatalo PI, Koivisto HM, Hietala JP, Puukka KS, Bloigu R, Niemelä OJ. Effect of moderate alcohol consumption on liver enzymes increases with increasing body mass index. Am JClin Nutr. 2008;88(4):1097-1103.

21. Naveau S, Giraud V, Borotto E, Aubert A, Capron F, Chaput JC. Excess weight risk factor for alcoholic liver disease. Hepatology. 1997;25(1):108-111.

22. Ruhl CE, Everhart JE. Joint effects of body weight and alcohol on elevated serum alanine aminotransferase in the United States population. Clin Gastroenterol Hepatol. 2005;3(12):1260-1268.

23. McGowan PM, et al. Notch1 inhibition alters the $\mathrm{CD} 44^{\text {hi }} / \mathrm{CD} 24^{\text {lo }}$ population and reduces the formation of brain metastases from breast cancer. Mol Cancer Res. 2011;9(7):834-844.

24. Raschke WC, Baird S, Ralph P, Nakoinz I. Functional macrophage cell lines transformed by Abelson leukemia virus. Cell. 1978;15(1):261-267.

25. Lowenstein CJ, et al. Macrophage nitric oxide synthase gene: two upstream regions mediate induction by interferon gamma and lipopolysaccharide. Proc Natl Acad Sci U S A. 1993;90(20):9730-9734.

26. Arteel GE, Iimuro Y, Yin M, Raleigh JA, Thurman RG. Chronic enteral ethanol treatment causes hypoxia in rat liver tissue in vivo. Hepatology. 1997;25(4):920-926.

27. Tsukamoto H, Xi XP. Incomplete compensation of enhanced hepatic oxygen consumption in rats with alcoholic centrilobular liver necrosis. Hepatology. 1989;9(2):302-306.

28. Tannahill GM, et al. Succinate is an inflammatory signal that induces IL-1 $\beta$ through HIF-1 $\alpha$. Nature. 2013;496(7444):238-242.

29. Rodríguez-Prados JC, et al. Substrate fate in activated macrophages: a comparison between innate, classic, and alternative activation. J Immunol. 2010;185(1):605-614.

30. Krawczyk CM, et al. Toll-like receptor-induced changes in glycolytic metabolism regulate dendritic cell activation. Blood. 2010;115(23):4742-4749.

31. Ferrick DA, Neilson A, Beeson C. Advances in measuring cellular bioenergetics using extracellular flux. Drug Discov Today. 2008;13(5-6):268-274.

32. Hill BG, Dranka BP, Zou L, Chatham JC, Dar-

ley-Usmar VM. Importance of the bioenergetic reserve capacity in response to cardiomyocyte stress induced by 4 -hydroxynonenal. Biochem $\mathrm{J}$. 2009;424(1):99-107.

33. Harris RA, Bowker-Kinley MM, Huang B, Wu P. Regulation of the activity of the pyruvate dehydrogenase complex. Adv Enzyme Regul. 2002;42:249-259.

34. Patel MS, Korotchkina LG. Regulation of the pyruvate dehydrogenase complex. Biochem Soc Trans. 2006;34(pt 2):217-222.

35. Morten KJ, Caky M, Matthews PM. Stabilization of the pyruvate dehydrogenase E1alpha subunit by dichloroacetate. Neurology. 1998;51(5):1331-1335.

36. Falkenberg M, Larsson NG, Gustafsson CM. DNA replication and transcription in mammalian mitochondria. Annu Rev Biochem. 2007;76:679-699.

37. Murphy MP, Smith RA. Targeting antioxidants to mitochondria by conjugation to lipophilic cations. Annu Rev Pharmacol Toxicol. 2007;47:629-656.

38. Bulua AC, et al. Mitochondrial reactive oxygen species promote production of proinflammatory cytokines and are elevated in TNFR1-associated periodic syndrome (TRAPS). J Exp Med. 2011;208(3):519-533.

39. West AP, et al. TLR signalling augments macrophage bactericidal activity through mitochondrial ROS. Nature. 2011;472(7344):476-480.

40. Freudenberg MA, Keppler D, Galanos C. Requirement for lipopolysaccharide-responsive macrophages in galactosamine-induced sensitization to endotoxin. Infect Immun. 1986;51(3):891-895.

41. Oh DY, Morinaga H, Talukdar S, Bae EJ, Olefsky JM. Increased macrophage migration into adipose tissue in obese mice. Diabetes. 2012;61(2):346-354.

42. Zigmond E, et al. Infiltrating monocyte-derived macrophages and resident kupffer cells display different ontogeny and functions in acute liver injury. J Immunol. 2014;193(1):344-353.

43. Rangarajan $\mathrm{A}$, et al. Notch signaling is a direct determinant of keratinocyte growth arrest and entry into differentiation. EMBO J. 2001;20(13):3427-3436.

44. Kodama Y, Hijikata M, Kageyama R, Shimotohno $\mathrm{K}$, Chiba $\mathrm{T}$. The role of notch signaling in the development of intrahepatic bile ducts. Gastroenterology. 2004;127(6):1775-1786.

45. Boni A, et al. Notch1 regulates the fate of cardiac progenitor cells. Proc Natl Acad Sci U S A. 2008;105(40):15529-15534.

46. Kitamura T, et al. A Foxo/Notch pathway controls myogenic differentiation and fiber type specifi- 
cation. J Clin Invest. 2007;117(9):2477-2485.

47. Kageyama R, Ohtsuka T. The Notch-Hes pathway in mammalian neural development. Cell Res. 1999;9(3):179-188.

48. Yao D, et al. Protein O-fucosyltransferase 1 (Pofut1) regulates lymphoid and myeloid homeostasis through modulation of Notch receptor ligand interactions. Blood. 2011;117(21):5652-5662.

49. O'Neill LA, Hardie DG. Metabolism of inflammation limited by AMPK and pseudo-starvation. Nature. 2013;493(7432):346-355.

50. MacMicking J, Xie QW, Nathan C. Nitric oxide and macrophage function. Annu Rev Immunol. 1997;15:323-350.

51. Riboldi E, Porta C, Morlacchi S, Viola A, Mantovani A, Sica A. Hypoxia-mediated regulation of macrophage functions in pathophysiology. Int Immunol. 2013;25(2):67-75.

52. Mukherjee T, Kim WS, Mandal L, Banerjee U. Interaction between Notch and Hif-alpha in development and survival of Drosophila blood cells. Science. 2011;332(6034):1210-1213.

53. Xu H, et al. Notch-RBP-J signaling regulates the transcription factor IRF8 to promote inflammatory macrophage polarization. Nat Immunol. 2012;13(7):642-650.

54. Olave I, Reinberg D, Vales LD. The mammalian transcriptional repressor RBP (CBF1) targets TFIID and TFIIA to prevent activated transcription. Genes Dev. 1998;12(11):1621-1637.

55. Xie QW, Kashiwabara Y, Nathan C. Role of transcription factor NF- $\mathrm{kB} / \mathrm{Rel}$ in induction of nitric oxide synthase. J Biol Chem. 1994;269(7):4705-4708.

56. Lendahl U, Lee KL, Yang H, Poellinger L. Generating specificity and diversity in the transcriptional response to hypoxia. Nat Rev Genet. 2009;10(12):821-832.

57. Han Z, Zhong L, Srivastava A, Stacpoole PW. Pyruvate dehydrogenase complex deficiency caused by ubiquitination and proteasome-mediated degradation of the E1 subunit. J Biol Chem.
2008;283(1):237-243.

58. Oren R, Farnham AE, Saito K, Milofsky E, Karnovsky ML. Metabolic patterns in three types of phagocytizing cells. J Cell Biol. 1963;17:487-501.

59. Voet D, Voet JG. Biochemistry. 2nd ed. Hoboken, New Jersey, USA: John Wiley \& Sons, Inc.; 1995.

60. Tavakoli S, Zamora D, Ullevig S, Asmis R. Bioenergetic profiles diverge during macrophage polarization: implications for the interpretation of 18F-FDG PET imaging of atherosclerosis. J Nucl Med. 2013;54(9):1661-1667.

61. Leeper-Woodford SK, Mills JW. Phagocytosis and ATP levels in alveolar macrophages during acute hypoxia. Am J Respir Cell Mol Biol. 1992;6(3):326-334.

62. Karnovsky ML, Lazdins J, Simmons SR. Metabolism of activated mononuclear phagocytes at rest and during phagocytosis. In: van Furth R, ed. Mononuclear Phagocytes in Immunity, Infection, and Pathology. 2nd ed. Oxford, United Kingdom: Blackwell Scientific Publications; 1975:423-438.

63. Stubbs M, Kühner AV, Glass EA, David JR, Karnovsky ML. Metabolic and functional studies on activated mouse macrophages. JExp Med. 1973;137(2):537-542.

64. Newsholme P. Why is L-glutamine metabolism important to cells of the immune system in health, postinjury, surgery or infection? J Nutr. 2001;131(9 suppl):2515S-2522S.

65. Perumalsamy LR, Nagala M, Sarin A. Notch-activated signaling cascade interacts with mitochondrial remodeling proteins to regulate cell survival. Proc Natl Acad Sci U S A. 2010;107(15):6882-6887.

66. Neupert W, Herrmann JM. Translocation of proteins into mitochondria. Annu Rev Biochem. 2007;76:723-749.

67. Lee SF, et al. $\gamma$-Secretase-regulated proteolysis of the Notch receptor by mitochondrial intermediate peptidase. J Biol Chem. 2011;286(31):27447-27453.

68. Wang H, et al. Genome-wide analysis reveals conserved and divergent features of Notch1/
RBPJ binding in human and murine T-lymphoblastic leukemia cells. Proc Natl Acad Sci US A. 2011;108(36):14908-14913.

69. Demehri S, et al. Notch-deficient skin induces a lethal systemic B-lymphoproliferative disorder by secreting TSLP, a sentinel for epidermal integrity. PLoS Biol. 2008;6(5):e123.

70. Morgan MJ, Liu ZG. Crosstalk of reactive oxygen species and NF-kB signaling. Cell Res. 2011;21(1):103-115.

71. Brunelle JK, et al. Oxygen sensing requires mitochondrial ROS but not oxidative phosphorylation. Cell Metab. 2005;1(6):409-414.

72. Guzy RD, et al. Mitochondrial complex III is required for hypoxia-induced ROS production and cellular oxygen sensing. Cell Metab. 2005;1(6):401-408.

73. Bedard K, Krause KH. The NOX family of ROS-generating NADPH oxidases: physiology and pathophysiology. Physiol Rev. 2007;87(1):245-313.

74. Arsenijevic D, et al. Disruption of the uncoupling protein-2 gene in mice reveals a role in immunity and reactive oxygen species production. Nat Genet. 2000;26(4):435-439.

75. Rousset S, Emre Y, Join-Lambert O, Hurtaud C, Ricquier D, Cassard-Doulcier AM. The uncoupling protein 2 modulates the cytokine balance in innate immunity. Cytokine. 2006;35(3-4):135-142.

76. Vogel RO, et al. Cytosolic signaling protein Ecsit also localizes to mitochondria where it interacts with chaperone NDUFAF1 and functions in complex I assembly. Genes Dev. 2007;21(5):615-624.

77. Pacher P, Beckman JS, Liaudet L. Nitric oxide and peroxynitrite in health and disease. Physiol Rev. 2007;87(1):315-424.

78. Clementi E, Nisoli E. Nitric oxide and mitochondrial biogenesis: a key to long-term regulation of cellular metabolism. Comp Biochem Physiol A Mol Integr Physiol. 2005;142(2):102-110.

79. Ueno A, Lazaro R, Wang PY, Higashiyama R, Machida K, Tsukamoto H. Mouse intragastric infusion (iG) model. Nat Protoc. 2012;7(4):771-781. 